\title{
¿Es útil el filtro para luz azul de los lentes intraoculares y aéreos para mejorar la salud visual? Una revisión sistemática de la literatura
}

\section{Is the blue light filter for spectacle and intraocular lenses helpful in improving ocular health? A systematic review of the literature}

\author{
L. Andrés Domínguez-Salgado ${ }^{\text {* }}$, Sebastián I. Chávez-Orta1 ${ }^{1}$ Miguel Á. Duque-Rodríguez", \\ Janette J. Franco-Contreras ${ }^{1}$, Daniel A. Herbert-Anaya ${ }^{1}$, María F. Montes-Rodríguez ${ }^{1}$, \\ Alejandro J. Zermeño-Arce ${ }^{2}$, María I. Patiño-López ${ }^{1}$, Mauricio Pierdant-Pérez ${ }^{1}$ y \\ Antonio A. Gordillo-Moscoso ${ }^{1}$
}

${ }^{1}$ Departamento de Epidemiología Clínica, Facultad de Medicina, Universidad Autónoma de San Luis Potosí, San Luis Potosí; ${ }^{2}$ Departamento de Retina y Vitreo, Instituto de Oftalmología Conde de Valenciana, Ciudad de México. México

\section{Resumen}

Introducción: La luz azul puede inducir daño retiniano. Hoy en día, los dispositivos electrónicos emiten altos niveles de este tipo de luz. Cerca del $90 \%$ de los usuarios experimentan síntomas como ojo seco, cefalea y visión borrosa. Algunos dispositivos oftalmológicos, como lentes aéreos e intraoculares, absorben la luz azul. Se han sugerido diversos beneficios y desventajas sobre su uso diario. Objetivo: Analizar si el uso de filtros de luz azul reduce el daño ocular inducido por este espectro de Iuz. Metodología: Se emplearon las siguientes bases de datos: PubMed, BVS, BIG, Academic Search Complete, Science Direct, Wiley Online Library, SpringerLink, Medic Latina, OvidSP, Trip Database, Clinical Key and UpToDate. Se realizó una recuperación secundaria para incrementar el número de estudios para su posterior análisis con la escala OPMER. Resultados: Diversos estudios demuestran que los filtros para lentes intraoculares tienen beneficios con pocos eventos adversos. En cuanto a los filtros para lentes aéreos, falta más investigación, pero parecen tener efectos beneficiosos. Conclusiones: Los estudios sugieren que no hay efectos perjudiciales en cuanto a desempeño visual con el uso de lentes intraoculares y pueden ser una opción para reducir el riesgo de desarrollar degeneración macular relacionada con la edad. Con el uso de filtros para lentes aéreos se observó una reducción en la fatiga ocular y pueden ayudar a mejorar la discapacidad visual. Es conocido que el uso de estos filtros in vitro minimiza el daño retiniano, pero la evidencia en humanos no es concluyente.

Palabras clave: Degeneración macular. Cambios maculares. Agudeza visual. Rendimiento visual. Filtro de luz azul.

\section{Abstract}

Introducción: Blue light can induce retinal damage. Nowadays technological devices emit high levels of this light. Up to $90 \%$ of users experience symptoms including eyestrain, headaches, ocular discomfort, dry eye and blurred vision. Some

Correspondencia:

*Luis Andrés Domínguez-Salgado

Av. Venustiano Carranza, 2405

Col. Lomas los Filtros

Fecha de recepción: 14-06-2019

Fecha de aceptación: 05-09-2019

E-mail: andres.lads@gmail.com

0187-4519/@ 2019 Sociedad Mexicana de Oftalmología. Publicado por Permanyer. Éste es un artículo open access bajo la licencia CC BY-NC-ND (http://creativecommons.org/licenses/by-nc-nd/4.0/).

Disponible en internet: 02-01-2020 Rev Mex Oftalmol. 2020;94(1):23-38 www.rmo.com.mx
cia CC BY-NC-ND 
ophthalmic devices like spectacle lenses and intraocular lenses (IOLs) absorb blue light. Benefits have been suggested as well as possible disadvantages. Objective: To analyze if the use of blue light filters reduces ocular damage produced from blue light spectrum. Method: The databases used were PubMed, BVS, BIG, Academic Search Complete, Science Direct, Wiley Online Library, SpringerLink, Medic Latina, OvidSP, Trip Database, Clinical Key and UpToDate. A second query was performed to increase the number of studies that were analyzed with the OPMER scale. Results: Several studies show that blue light filter IOLs have benefits in ocular health with low adverse effects. For other ophthalmic devices like spectacle lenses, evidence is lacking. Nevertheless, it seems to be beneficial. Conclusions: The studies suggest there are no significant harmful effects of blue-blocking IOLs on visual performance and could be an option to reduce the risk of development of age-related macular degeneration. With the use of spectacle lenses there was a reduction in eye fatigue, suggesting it may help to ameliorate visual impairment. It is known that the use of blue light filters in vitro minimizes the damage to retinal cells but data in humans is inconclusive, especially regarding spectacle lenses.

Key words: Macular degeneration. Macular changes. Visual acuity. Visual performance. Blue light filters.

\section{Introducción}

La utilidad de los lentes con filtro de luz azul para prevenir la fototoxicidad retiniana sigue siendo incierta. Desde la década de 1980 , se ha especulado que la luz dentro del espectro visible de la longitud de onda azul, así como la luz ultravioleta, causan daños en múltiples estructuras oculares. Por esta razón, era necesario agregar filtros específicos a los lentes intraoculares (LIO) ${ }^{1,2}$.

La luz azul tiene una alta energía fotoquímica e induce apoptosis celular en las células del epitelio pigmentario de la retina (EPR) debido a su fototoxicidad; el riesgo para la retina por la estimulación con luz azul ha sido bien demostrado utilizando fuentes de luz de alta intensidad. Hoy en día, nuestros dispositivos tecnológicos con pantallas que contienen diodos emisores de luz (LED) y lámparas fluorescentes compactas emiten niveles relativamente altos de luz azul. Si bien varios estudios han demostrado que los LIO que bloquean la luz dentro del espectro azul tienen múltiples beneficios para mejorar la salud ocular con desventajas mínimas, existen muy pocos estudios sobre anteojos con filtro azul como factor de protección, y este tipo de filtros puede ser significativamente más caro que los lentes que no los incluyen ${ }^{1,2}$.

Las longitudes de onda azules del espectro electromagnético $(400-500 \mathrm{~nm})$ pueden provocar daños fototóxicos en la retina. Con el aumento de la edad, la exposición durante toda la vida de las células del EPR a la luz, incluso con la intensidad diaria, puede tener un impacto significativo en su degeneración. Los eventos celulares y moleculares que subyacen al daño fotoquímico de la retina por la luz, incluida la apoptosis de fotorreceptores, se han analizado en modelos animales experimentales. Se han descrito dos tipos de daño retiniano inducido por la luz. Noell, et al. explicaron el daño retiniano de clase I causado por bajos niveles de luz y exposiciones prolongadas. La extensión del daño observado está relacionada con la duración de la exposición y la longitud de onda de la luz utilizada, con una longitud de onda más corta (luz azul) que tiene un mayor efecto. El daño fotoquímico de clase II o el riesgo de la luz azul, descrito por Ham, et al., se relaciona con exposiciones retinianas cortas de alta intensidad. En este caso, la toxicidad disminuye al tiempo que aumenta la longitud de onda de los rayos ultravioleta (UV) a través de la parte violeta y azul del espectro visible. El daño retiniano está relacionado con el tiempo y la intensidad de la exposición, además de la longitud de onda, con un pico de excitación de alrededor de $440 \mathrm{~nm}^{1,2}$.

Tanto los estudios en humanos como en animales sugieren que son principalmente las longitudes de onda cortas dentro de los rangos de luz UV y azul las que provocan daños en la retina e inducen toxicidad fotoquímica o estrés oxidativo al EPR, con la formación de especies reactivas de oxígeno ${ }^{3,4}$.

La luz azul es emitida naturalmente por el sol y ayuda a regular la melatonina en el cerebro, activando el ciclo natural de sueño-vigilia. Sin embargo, la tecnología ha alterado esto, ya que la mayoría de los dispositivos electrónicos emiten luz azul. Hoy en día, hasta el $90 \%$ de los usuarios de computadoras y otros dispositivos electrónicos digitales experimentan síntomas que incluyen fatiga visual, cefalea, malestar ocular, ojo seco, diplopía y visión borrosa. A pesar de los riesgos, la radiación espectralmente ponderada de estos dispositivos no alcanza los límites de exposición internacionales, incluso con una visualización prolongada; sin embargo, esto no ha sido bien estudiado. Moon, et al. demostraron que los dispositivos con un pico de luz azul a una longitud de onda más corta causan una disminución más pronunciada del número de células. El daño de la luz como posible factor contribuyente al desarrollo de la degeneración macular 
Tabla 1. Palabras clave y términos

\begin{tabular}{|c|c|c|c|c|}
\hline Palabras clave & $\begin{array}{l}\text { Términos } \\
\text { MeSH }\end{array}$ & Sinónimos & Términos DeCS & Sinónimos \\
\hline $\begin{array}{l}\text { Degeneración } \\
\text { macular }\end{array}$ & $\begin{array}{l}\text { Degeneración } \\
\text { macular }\end{array}$ & $\begin{array}{l}\text { Cambios maculares } \\
\text { Degeneración macular relacionada con la edad } \\
\text { Maculopatías relacionadas con la edad } \\
\text { Maculopatía relacionada con la edad } \\
\text { Degeneración macular, relacionada con la edad } \\
\text { Distrofia macular } \\
\text { Maculopatías, relacionadas con la edad } \\
\text { Maculopatía, relacionada con la edad }\end{array}$ & $\begin{array}{l}\text { Degeneración } \\
\text { macular }\end{array}$ & $\begin{array}{l}\text { Maculopatía relacionada } \\
\text { con la edad }\end{array}$ \\
\hline Agudeza visual & $\begin{array}{l}\text { Agudeza } \\
\text { visual }\end{array}$ & Desempeño visual & Agudeza visual & $\begin{array}{l}\text { Acuidad visual } \\
\text { Desempeño visual }\end{array}$ \\
\hline $\begin{array}{l}\text { Filtros de luz } \\
\text { azul }\end{array}$ & $\begin{array}{l}\text { Título/ } \\
\text { Resumen }\end{array}$ & $\begin{array}{l}\text { Filtros de luz azul } \\
\text { Lentes para luz azul }\end{array}$ & Filtro de luz azul & $\begin{array}{l}\text { Lentes con filtro para luz } \\
\text { azul }\end{array}$ \\
\hline
\end{tabular}

relacionada con la edad (DMRE) se sugirió por primera vez en la década de 1980. En el estudio de la Bahía de Chesapeake, hubo una relación entre los altos niveles de exposición a la luz azul y visible y el desarrollo de DMRE, especialmente más tarde, en etapas avanzadas de la vida ${ }^{5-9}$.

Las propiedades de los medios oculares limitan la transmisión de ciertas longitudes de onda. La córnea absorbe la radiación de longitudes de onda inferiores a $300 \mathrm{~nm}$, pero permite la transmisión de radiación con longitudes de onda de entre 300 y $400 \mathrm{~nm}$. A medida que el cristalino envejece, acumula cromóforos amarillos, lo que resulta en una reducción constante en la transmisión de la luz visible de longitud de onda corta. En los ojos afáquicos o pseudofáquicos, la radiación azul y UV Ilega directamente a la retina, por lo que en los últimos años han surgido medidas profilácticas contra la DMRE. Esto dio como resultado la implantación de LIO «amarillas» que absorben la radiación azul de alta energía y pueden ser más adecuadas para casos que requieren protección especial de la retina ${ }^{10-13}$.

Algunos beneficios sugeridos incluyen protección contra el daño de la retina, con un posible papel en la prevención del desarrollo o exacerbación de la DMRE, la mejora de la sensibilidad al contraste (SC), la reducción del glare en condiciones fotópicas y mesópicas y la reducción de la alteración en la visión del color azul. Las posibles desventajas incluyen alteraciones de la percepción del color, disminución de la sensibilidad escotópica e interrupción de la sincronización del sistema circadiano. Además, también se ha afirmado que los anteojos con filtro azul mejoran la calidad del sueño después del uso de dispositivos electrónicos por la noche, y reducen la fatiga ocular y los síntomas de fatiga ocular durante el trabajo intenso en la computadora. Los fabricantes de anteojos que bloquean la luz azul afirman que alivian las molestias oculares debido al uso de dispositivos digitales, mejoran la calidad del sueño, la fatiga visual y mejoran la protección de la retina. Hoy en día, el uso de dispositivos digitales es más que necesario en sitios como escuelas, lugares de trabajo y entornos domésticos, donde pasamos varias horas al día usándolos ${ }^{14-18}$.

El objetivo de este estudio es analizar sistemáticamente si el uso de filtros de luz azul reduce el daño ocular producido por el espectro de luz de longitud de onda corta, para ayudar a nuestros colegas a tomar decisiones sobre la prescripción de este tipo de anteojos considerando los beneficios y riesgos para cada paciente.

\section{Métodos}

\section{Estrategia de búsqueda}

Se realizó una estrategia de búsqueda en diferentes bases de datos durante el periodo de enero a febrero de 2019; utilizando las palabras clave, los términos $\mathrm{MeSH}$ y finalmente los términos DeCS descritos en la tabla 1.

\section{Bases de datos y fuentes de información}

Las principales bases de datos y motores de metabúsqueda utilizados en este artículo fueron: PubMed, Biblioteca Virtual en Salud (BVS), Búsqueda de Información Global (BIG), Academic Search Complete, Science Direct, Wiley Online Library, SpringerLink, Medic Latina, Wolters Kluwer Health 


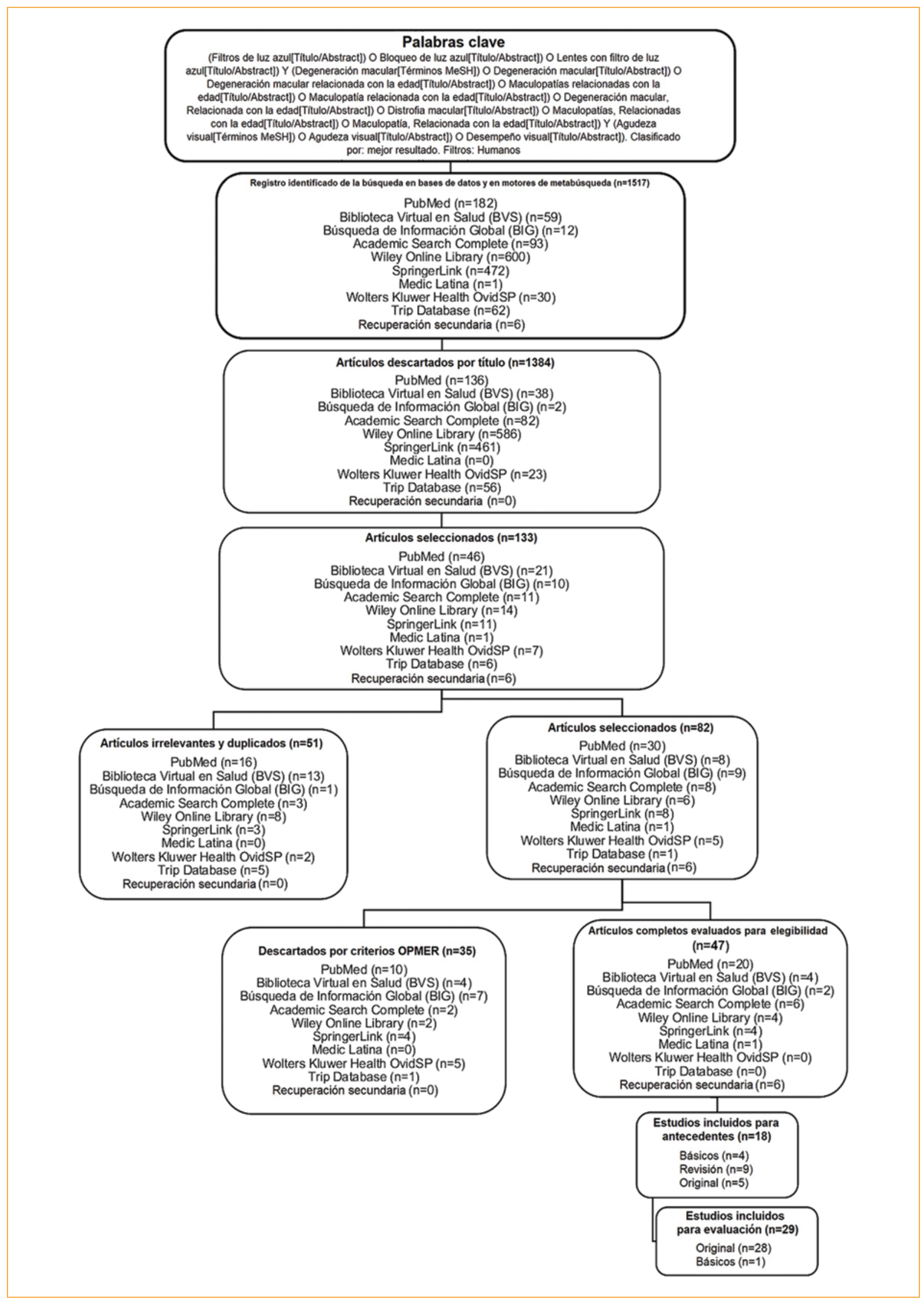

Figura 1. Estrategia de búsqueda y metodología de bases de datos. 
OvidSP, Trip Database, Clinical Key y UpToDate. También hicimos una segunda búsqueda para aumentar el número de estudios. Con todas las palabras clave, los términos MeSH y los términos DeCs, creamos tres universos diferentes vinculados con el operador booleano "Y», también en cada uno de ellos el operador booleano utilizado para vincular los términos MeSH con sus sinónimos era "O». Consulte la figura 1 para consultar la estrategia de búsqueda completa.

\section{Límites}

En bases de datos como PubMed, Biblioteca Virtual en Salud (BVS) y Academic Search Complete que son multidisciplinarias, utilizamos el filtro "estudios en humanos"; con los otros motores de metabúsqueda no se necesitaban filtros.

\section{Criterios de selección}

Primero, uno de los autores seleccionó los artículos por títulos considerando los que tienen una gran cantidad de palabras clave. Luego, todos los autores revisaron de forma independiente los resúmenes. Finalmente, uno de los autores analizó los estudios potencialmente relevantes con versiones de texto completo utilizando una escala de calidad para tomar una decisión final sobre la inclusión. La evidencia de los artículos originales se evaluó con la escala OPMER. Esta escala fue desarrollada en el Departamento de Epidemiología de la Universidad Autónoma de San Luis Potosí, se basa en los criterios PRISMA y contiene una lista de verificación que incluye cinco grupos de validez que abordan la solidez científica de los artículos originales.

- Objetivo

- Población

- Metodología

- Estadística

- Resultados

Se seleccionaba un artículo si obtenía más de 14 puntos.

\section{Criterios de exclusión}

Se excluyeron todos los estudios con un título no relacionado con el tema principal. Finalmente, después de leer los artículos seleccionados, si la escala OPMER estaba por debajo de 14 puntos, el estudio era excluido.

\section{Desarrollo del tema}

Veintinueve artículos originales cumplieron con los criterios y fueron seleccionados como los más importantes y confiables (Tabla 2). Sus resultados se presentan a continuación.

\section{Discusión}

Para facilitar el análisis de los documentos y compararlos entre grupos, decidimos distribuir los artículos en dos grupos: uno sobre LIO y sus efectos y el otro sobre anteojos.

Owczarek, et al. compararon la cantidad de transmisión de luz de dos tipos de LIO acrílicos hidrofóbicos con el mismo poder de refracción de 20.0 dioptrías, uno que contiene un cromóforo amarillo $\left(\right.$ AcrySof ${ }^{\circledR} \mathrm{Na}-$ tural SN60AT Alcon Labs, EE. UU.) y otro sin un cromóforo (Alcon Labs). Se evaluó la transmisión de la luz, en condiciones de luz del día y nocturna. Los resultados muestran un valor significativamente más bajo de transmisión de la luz $(74.41 \%$ y $92.14 \%$, respectivamente) para los LIO con filtro de luz azul en comparación con los LIO no modificados, respectivamente. Por otro lado, parece razonable sugerir que bloquear la radiación de luz azul en condiciones mesópicas puede mejorar la calidad visual ${ }^{19}$.

En 2006, se diseñó un estudio prospectivo aleatorizado para comparar los resultados visuales postoperatorios: mejor agudeza visual (AV) corregida, SC y percepción del color con los LIO AcrySof ${ }^{\circledR}$ Natural (SN60AT) y AcrySof ${ }^{\circledR}$ de una sola pieza (SA60AT) que filtran la luz azul. El grupo de SN60AT incluyó nueve ojos de nueve pacientes, y el grupo de SA60AT incluyó diez ojos de diez pacientes. La evaluación postoperatoria incluyó un examen clínico completo a los 1,3 y 6 meses después de la cirugía, incluida la mejor AV corregida (cartilla de Snellen), la SC (cartilla de sensibilidad al contraste de Pelli-Robson) y la percepción del color (prueba de Farnsworth-Munsell D-15), medidas a los 1, 3 y 6 meses después de la cirugía. Ambos grupos mostraron una mejora en las puntuaciones logarítmicas de SC $(p=0.08)$ en condiciones mesópicas y fotópicas, y la percepción del color no mostró diferencias estadísticamente significativas entre los grupos ${ }^{20}$.

Se ha informado que los pacientes diabéticos desarrollan alteraciones de la visión del color. Estudios previos sugieren que el sistema de conos sensibles a longitudes de onda cortas, o conos S, son más vulnerables a la diabetes. Teóricamente, el uso de filtros amarillos puede aumentar el desempeño visual en este 
Tabla 2. Resultados de artículos

\begin{tabular}{|c|c|c|c|c|c|c|}
\hline Título & Autor & Año & $\begin{array}{l}\text { Tamaño de } \\
\text { la muestra }\end{array}$ & Objetivo & $\begin{array}{l}\text { Tipo de } \\
\text { artículo }\end{array}$ & OPMER \\
\hline $\begin{array}{l}\text { Blue-Light Filtering Spectacle } \\
\text { Lenses: Optical and Clinical } \\
\text { Performances. }\end{array}$ & Leung et al. & 2017 & 80 & $\begin{array}{l}\text { Evaluar el desempeño óptico de los } \\
\text { anteojos con filtro de luz azul e } \\
\text { investigar si una reducción en la } \\
\text { transmisión de luz azul afecta el } \\
\text { rendimiento visual y la calidad del sueño }\end{array}$ & Original & 18 \\
\hline $\begin{array}{l}\text { Short-Wavelength } \\
\text { Light-Blocking Eyeglasses } \\
\text { Attenuate Symptoms of Eye } \\
\text { Fatigue }\end{array}$ & Lin JB et al. & 2017 & 36 & $\begin{array}{l}\text { Determinar si los sujetos que usan } \\
\text { anteojos que bloquean la longitud de } \\
\text { onda corta durante el trabajo en la } \\
\text { computadora exhiben menos fatiga } \\
\text { visual e informan menos síntomas de } \\
\text { incomodidad visual que los sujetos } \\
\text { que usan anteojos transparentes }\end{array}$ & Original & 17 \\
\hline $\begin{array}{l}\text { Visual function improvement } \\
\text { using photocromic and } \\
\text { selective blue-violet light } \\
\text { filtering spectacle lenses in } \\
\text { patients affected by retinal } \\
\text { diseases }\end{array}$ & Colombo et al. & 2017 & 60 & $\begin{array}{l}\text { Evaluar parámetros visuales } \\
\text { funcionales mediante el uso de } \\
\text { anteojos con filtrado fotocromático y } \\
\text { selectivo de luz azul-violeta en } \\
\text { pacientes afectados por un escotoma } \\
\text { central o periférico debido a } \\
\text { enfermedades de la retina }\end{array}$ & Original & 16 \\
\hline $\begin{array}{l}\text { Psychophysical } \\
\text { Measurements of Luminance } \\
\text { Contrast Sensitivity and Color } \\
\text { Discrimination with } \\
\text { Transparent and Blue-Light } \\
\text { Filter Intraocular Lenses }\end{array}$ & Da Costa et al. & 2017 & 15 & $\begin{array}{l}\text { Medir la sensibilidad al contraste de } \\
\text { luminancia y umbrales de visión al } \\
\text { color en sujetos normales que utilizan } \\
\text { un lente con filtro de luz azul y material } \\
\text { transparente de lente intraocular }\end{array}$ & Original & 15 \\
\hline $\begin{array}{l}\text { Sleep and mood changes in } \\
\text { advanced age after } \\
\text { blue-blocking (yellow) intra } \\
\text { ocular lens (IOLs) } \\
\text { implantation during cataract } \\
\text { surgical treatment: a } \\
\text { randomized controlled trial. }\end{array}$ & Zambrowski et al. & 2017 & 204 & $\begin{array}{l}\text { Demostrar la superioridad de los } \\
\text { lentes intraoculares (LIO) amarillos en } \\
\text { comparación con los LIO } \\
\text { transparentes sobre el sueño y los } \\
\text { cambios de humor antes y después de } \\
\text { la cirugía de catarata bilateral }\end{array}$ & Original & 16 \\
\hline $\begin{array}{l}\text { Protective effect of blue-light } \\
\text { shield eyewear for adults } \\
\text { against light pollution from } \\
\text { self-luminous devices used at } \\
\text { night }\end{array}$ & Ayaki et al. & 2016 & 12 & $\begin{array}{l}\text { Explorar si los anteojos con filtro de luz } \\
\text { azul pueden proteger contra los efectos } \\
\text { de la contaminación por la luz azul de } \\
\text { los dispositivos portátiles } \\
\text { autoluminiscentes utilizados por la noche }\end{array}$ & Original & 16 \\
\hline $\begin{array}{l}\text { The effect of blue-blocking } \\
\text { and neutral intraocular lenses } \\
\text { on circadian photoentrainment } \\
\text { and sleep one year after } \\
\text { cataract surgery }\end{array}$ & Brøndsted et al. & 2016 & 67 & $\begin{array}{l}\text { Comparar el efecto a largo plazo en el } \\
\text { fotoarrastre circadiano y el sueño en } \\
\text { pacientes implantados con lentes } \\
\text { intraoculares neutrales y con filtro } \\
\text { azul } 1 \text { año después de la cirugía de } \\
\text { catarata }\end{array}$ & Original & 15 \\
\hline $\begin{array}{l}\text { Reducing Short-Wavelength } \\
\text { Blue Light in Dry Eye Patients } \\
\text { with Unstable Tear Film } \\
\text { Improves Performance on } \\
\text { Tests of Visual Acuity. }\end{array}$ & Kaido et al. & 2016 & 40 & $\begin{array}{l}\text { Investigar si la supresión de la luz azul } \\
\text { puede mejorar la función visual en } \\
\text { pacientes con ojo seco con } \\
\text { disminución del tiempo de ruptura de } \\
\text { la película lagrimal }\end{array}$ & Original & 17 \\
\hline $\begin{array}{l}\text { Effect of blue light-filtering } \\
\text { intraocular lens on color } \\
\text { vision in patients with } \\
\text { macular diseases after } \\
\text { vitrectomy }\end{array}$ & Mokuno et al. & 2016 & 67 & $\begin{array}{l}\text { Evaluar la visión al color de los } \\
\text { pacientes con enfermedades } \\
\text { maculares después de implantar una } \\
\text { lente intraocular (LIO) con filtro de luz } \\
\text { azul durante la vitrectomía. }\end{array}$ & Original & 16 \\
\hline $\begin{array}{l}\text { Light transmission through } \\
\text { intraocular lenses with or } \\
\text { without yellow chromophore } \\
\text { (blue light filter) and its } \\
\text { potential influence on } \\
\text { functional vision in everyday } \\
\text { environmental conditions }\end{array}$ & Owczarek et al. & 2016 & - & $\begin{array}{l}\text { Analizar la posible influencia de la } \\
\text { transmisión de la luz a través de } \\
\text { lentes intraoculares con o sin } \\
\text { cromóforo amarillo sobre la visión } \\
\text { funcional en entornos cotidianos }\end{array}$ & Original & 15 \\
\hline
\end{tabular}


Tabla 2. Resultados de artículos (continuación)

\section{Título A \\ Blue light-filtering intraocular lenses and post-operative mood: a pilot clinical study \\ Effect of yellow filter on visual acuity and contrast sensitivity under glare condition among different age groups}

Nuevos lentes solares con certificado de seguridad retiniana: análisis de la función visual mediante la valoración de agudeza visual y estereoscópica, discriminación del color y sensibilidad al contraste

Effect of Blue Light-Reducing Eye Glasses on Critical Flicker Frequency

Espesor macular medido con tomografía de coherencia óptica en ojos pseudoafáquicos con implante amarillo vs. transparente

Do blue-light filtering intraocular lenses affect visual function?

Short wavelength light filtering by the natural human lens and IOLs - implications for entrainment of circadian rhythm

Influence of

blue-light-filtering intraocular lenses on daytime levels of melatonin (BluMel-Study)

Effects of blue light-filtering intraocular lenses on the macula, contrast sensitivity, and color vision after a long-term follow-up

Effects of yellow filters on visual acuity, contrast sensitivity and reading under conditions of forward light scatter
Mahjoob et al.

2015

60

Bonnin Arias et al.

2015

36

Ide et al.

Chamorro et al.

Brøndsted et al.

Kubista et al.

Kara-Junior et al.

\section{Objetivo}

Determinar si la implantación de LIO con filtro azul afecta el estado de ánimo postoperatorio, induciendo más depresión, en comparación con los pacientes sometidos a implante de LIO

convencionales

Investigar el efecto del filtro amarillo sobre la agudeza visual y la sensibilidad al contraste bajo condiciones de glare en diferentes edades

Comparar el efecto sobre la función visual de las nuevas lentes solares con certificado de seguridad retiniana versus lentes solares tradicionales

Evaluar el efecto de bloquear la luz de longitud de onda corta sobre la frecuencia de parpadeo de fusión crítica (CFF)

Evaluar mediante tomografía de coherencia óptica (OCT) las variaciones del grosor de las células maculares en el tiempo en pacientes pseudofáquicos implantados con un LIO transparente en comparación con su ojo contralateral respectivo implantado con LIO amarillos

Evaluar diferentes aspectos de la función visual, los cambios maculares y las diferencias subjetivas entre el ojo con un LIO con filtro de luz ultravioleta (UV) y azul y el otro ojo con un LIO con filtro de luz UV

Examinar el efecto del cristalino humano envejecido sobre el fotoarrastre del ritmo circadiano, y comparar con los LIO diseñados para bloquear la radiación UV, la luz violeta 0 azul

Analizar la influencia de los LIO con filtros de luz azul sobre los niveles diurnos de melatonina in vivo

Evaluar los posibles efectos secundarios y la posible protección 5 años después de la implantación de un LIO con un filtro de luz azul (de color amarillo)

Comparar los efectos de tres filtros fotocromáticos de Corning (CPF, 450, 511 y 527) sobre la agudeza visual y la sensibilidad al contraste con y sin glare y al leer sin glare bajo condiciones de dispersión de luz, para determinar cuál proporcionó el efecto más beneficioso en estos aspectos de la función visual
Original 15

Original

\begin{tabular}{|c|r|}
\hline Tipo de & OPMER \\
\hline articulo & \\
\hline Original & 17 \\
\hline Original & 16 \\
\hline Original & \\
\hline Original & \\
\hline Original & \\
\hline & \\
\hline & \\
\hline & \\
\hline
\end{tabular}


Tabla 2. Resultados de artículos (continuación)

\section{Título \\ intraocular lenses on retinal \\ nerve fiber layer \\ measurements by \\ spectral-domain optical \\ coherence tomography}

Influence of blue light-filtering Kim et al.

Autor

Contralateral comparison of

Hammond et al.

2010

52

non-blue-filtering intraocular

lenses: glare disability,

heterochromatic contrast,

and photo stress recovery

Amber lenses to block blue

light and improve sleep: a

Augmentation of macular pigment following

implantation of blue

light-filtering intraocular

lenses at the time of cataract surgery

Visual function and performance with blue-light blocking filters in age-related macular degeneration

Blue blocker glasses impede the capacity of bright light to suppress melatonin production

Visual outcomes with the yellow intraocular lens

Blue-light filtering intraocular lens in patients with diabetes: contrast sensitivity and chromatic discrimination

Burkhart y Phelps

2009

20

Nolan et al.

Kiser et al.

Leibovitch et al. blue-filtering and

randomized trial

\section{Objetivo}

Evaluar la influencia de los LIO con filtro de luz azul en las mediciones del grosor de la capa de fibras nerviosas peripapilares de la retina por OCT de dominio espectral

Comparar el desempeño visual en ojos visible

Demostrar cambios en la calidad general del sueño y efecto positivo/ negativo

\section{Investigar si las propiedades de} filtrado de luz azul del lente intraocular AcrySof Natural de Alcon (ANIOL) implantado durante la cirugía de catarata, afecta la densidad óptica del pigmento macular

Determinar si un filtro de luz azul afectaría el desempeño durante la coordinación ojo-mano y las tareas de movilidad bajo iluminación escotópica, bajo sensibilidad escotópica medida psicofísicamente o discriminación de color en pacientes con degeneración macular relacionada con la edad

\section{Sasseville et al. $\quad 2006 \quad 14$}

Determinar la capacidad de los filtros azules para prevenir la supresión de melatonina durante un pulso de luz de 60 minutos a $4000 \mathrm{~lx}$, que es una intensidad que se sabe que es suficiente para inducir la supresión máxima de melatonina

Comparar la mejor agudeza visual a distancia postoperatoria, la sensibilidad al contraste y la percepción de colores con los LIO AcrySof Natural (SN60AT) y AcrySof de una pieza (SA60AT) con filtro de luz azul

Evaluar los posibles cambios en la sensibilidad al contraste y la discriminación de color en pacientes diabéticos que se sometieron a cirugía de cataratas e implante de LIO AcrySof Natural (SN60AT) con filtro de luz azul en comparación con un LIO con filtro de luz ultravioleta (AcrySof SA60AT).

The effects of coloured light filter overlays on reading rates in age-related macular degeneration
Eperjesi
12 superposición de filtros de luz de colores sobre la velocidad de lectura de personas con degeneración LIO que filtran la luz azul de onda corta versus los ojos contralaterales con LIO que no filtran la luz azul

\section{Tipo de \\ OPMER} artículo

Original

15

Original

Original

Original

Original

Original

Determinar el efecto de la

Original 14 macular relacionada con la edad 
grupo de pacientes. Rodríguez, et al. evaluaron los posibles cambios en la SC y la discriminación de colores en pacientes diabéticos que se sometieron a cirugía de catarata e implante de LIO AcrySof Natural SN60AT con filtro de luz azul en comparación con un LIO AcrySof SA60AT con filtro de luz UV. Se reclutaron 44 ojos de 22 pacientes diabéticos en un estudio de control con los ojos contralaterales implantados con un filtro de luz azul. Los pacientes recibieron AcrySof Natural en 1 ojo y AcrySof SA60AT en el ojo contralateral. Tres meses después de la cirugía, la función de SC (FSC) monocular se midió con la cartilla de sensibilidad al contraste CSV 1000-E a distancia, y la discriminación de colores se analizó con la prueba de 100 tonos de Farnsworth-Munsell. No se observaron diferencias estadísticamente significativas entre los LIO ( $p=0.62)$. Los ojos implantados con los LIO con filtro de luz azul mostraron mejores valores de SC que los ojos implantados con LIO sin color amarillo $(p<0.05)$. Los LIO con filtro de luz azul no modificaron la discriminación cromática en comparación con los LIO sin color amarillo ( $p=0.62)$. En pacientes diabéticos, el LIO AcrySof Natural proporciona una mejor SC que el AcrySof SA60AT. El filtro de luz azul del LIO AcrySof Natural no causó defectos de discriminación cromática con base en los puntajes de error totales, y mejoró la visión del color en el eje cromático azul-amarillo en pacientes diabéticos ${ }^{21}$.

Hammond, et al., en 2010, compararon el desempeño visual en ojos con LIO que filtran luz azul versus ojos contralaterales con LIO que no filtran la luz azul visible. Fueron evaluados 52 sujetos, y se encontró que la media de discapacidad por glare fue significativamente menor $(p=0.04)$ en el grupo de LIO con filtro azul que en el grupo control, así como que el umbral de contraste heterocromático fue significativamente mayor $(p=0.0003)$. Además, la recuperación del fotoestrés fue más rápida $(p=0.02)$ en el grupo de LIO con filtro azul, con una recuperación de la función visual 5 segundos más rápido (esto se traduce en 440 pies cuando se conduce a 60 millas por hora) ${ }^{22}$.

Da Costa, et al. evaluaron la SC de 15 participantes. Compararon la medida de SC del filtro para luz transparente y azul, y encontraron que los sujetos tenían una mayor SC con el filtro transparente en comparación con el filtro azul $(p=0.632)$. Sin embargo, los participantes $(87 \%)$ refirieron tener una visión más cómoda con el lente con filtro para luz azul $(p=0.016)$. La visión del color tuvo una significancia estadísticamente más alta para el filtro de luz azul $(p=0.003)$. La impresión subjetiva mostró en todos los participantes una mejor visión de color en condiciones de filtro de luz azul. En resumen, no se observaron diferencias cuantitativas en la sensibilidad a la luminancia entre ambos tipos de lentes, aunque se informó que el filtro de luz azul fue más cómodo que el filtro transparente ${ }^{23}$.

En $2011 \mathrm{Kara} \mathrm{Jr}$, et al. evaluaron los posibles efectos secundarios y la posible protección de un LIO con un filtro de luz azul. Los pacientes recibieron aleatoriamente un LIO con filtro UV y para luz azul (AcrySof Natural SN60AT) en un ojo y un LIO acrílico que filtra la luz UV (AcrySof SA60AT) en el otro ojo. Midieron la SC, la visión de colores y los hallazgos maculares 5 años después de la cirugía en condiciones fotópicas y escotópicas, y se realizó un examen macular detallado. No hubo hallazgos significativos mediante tomografía de coherencia óptica ni clínicos en términos de DMRE, o diferencias estadísticamente significativas del grosor macular central entre los 2 grupos ( $p=0.712)$. Además, no hubo diferencias significativas bajo condiciones fotópicas o escotópicas en ninguna frecuencia espacial estudiada, ni cambios en la discriminación de colores $(p=0.674)$. La ventaja potencial del LIO con filtro para proporcionar protección a las células maculares, sigue sin estar clara ${ }^{24}$.

En 2014, Lavric, et al. desarrollaron un estudio para evaluar el efecto de un LIO con filtro para luz azul sobre la función visual, los cambios maculares y la calidad visual subjetiva. En un ojo, se implantó un AcrySof SA60AT (un LIO con filtro de luz UV), mientras que en el ojo contralateral, se implantó un AcrySof IQ SN60WF (un LIO con filtro para luz azul). Cada paciente se sometió a pruebas de AV, pruebas de visión de colores (pruebas de Ishihara y de 100 tonos de Farnsworth Munsell) y pruebas de SC. La mácula se evaluó mediante tomografía de coherencia óptica y examen clínico, y la calidad visual subjetiva se evaluó con el Cuestionario de funcionamiento visual del National Eye Institute. Con respecto a la visión de colores, no se detectaron cambios significativos en las puntuaciones de error de Ishihara y de 100 tonos de Farnsworth-Munsell entre ambos ojos ( $p=0.48$ y $p=0.59$, respectivamente). El análisis de SC no mostró diferencias significativas entre los grupos. Además, no se observaron diferencias estadísticamente significativas en el grosor macular central y el volumen macular total ( $p=0.72$ y $p=0.61$, respectivamente). Este estudio no mostró efectos significativos de un LIO con filtro para luz azul sobre la AV, la percepción de colores y la $\mathrm{SC}^{25}$.

En 2016, Mokuno, et al. realizaron un estudio para evaluar la visión de colores de pacientes con enfermedades maculares después de implantar un LIO con filtro para luz azul. Se utilizó la prueba de 100 tonos de Farnsworth-Munsell para determinar las puntuaciones 
de error total (PET) y la media de las puntuaciones de error en condiciones fotópicas y mesópicas en ambos grupos. Las PET en condiciones mesópicas fueron significativamente mayores que en condiciones fotópicas en ambos grupos $(p<0.05)$. Sin embargo, las PET en el grupo de enfermedad macular no fueron significativamente diferentes en comparación con el grupo de enfermedad no macular en condiciones fotópicas y mesópicas. Las medias de las puntuaciones de error en condiciones fotópicas fueron significativamente más altas en el grupo de enfermedad macular en comparación con el grupo de enfermedad no macular. En condiciones mesópicas, la media de las puntuaciones de error fue significativamente más alta en el grupo de enfermedad no macular que en el grupo de enfermedad macular $(p<0.05)$. Estos resultados indican que los LIO que filtran la luz azul no alteran la discriminación de colores en los ojos con enfermedades maculares ${ }^{26}$.

Kiser, et al. en 2008 estudiaron si un filtro para luz azul afecta la sensibilidad retiniana escotópica y el desempeño visual (movilidad, coordinación ojo-mano) en pacientes con DMRE temprana. Las mediciones escotópicas realizadas con y sin un filtro de atenuación de la luz azul incluyeron un curso de obstáculos para movilidad, la manipulación de bloques cilíndricos y una prueba con flash de campo completo con adaptación psicofísica a la obscuridad. Una tarea de clasificación del color de un calcetín azul marino y azul evaluó la discriminación fotópica de colores. Con el filtro, en promedio, hubo un aumento del $13 \%$ en el tiempo durante la prueba de bloque. Las diferencias en el tiempo y el número de topes con versus sin el filtro no fueron significativas para el curso de movilidad. El desempeño con y sin filtro se correlacionó bien para los bloques $(r=0.70)$, la prueba de flash $(r=0.83)$ y la movilidad $(r=0.66)$, y las pendientes de regresión no fueron significativamente diferentes de la unidad. El $77 \%$ de los sujetos identificaron erróneamente al menos un calcetín azul marino como negro con el filtro en comparación con el $9 \%$ sin él, con un aumento significativo en tales identificaciones erróneas con el filtro. La diferencia en la función o el desempeño visual escotópico con un filtro para luz azul versus sin un filtro para luz azul probablemente no ocasione un efecto o riesgo clínicamente significativo; sin embargo, la detección del color azul marino puede verse afectada ${ }^{27}$.

Brønsted, et al. examinaron el efecto del envejecimiento del cristalino humano sobre el fotoarrastre del ritmo circadiano, y lo compararon con los LIO diseñados para bloquear la radiación UV, la luz violeta o azul. Se calculó el potencial de fotoarrastre del ritmo circadiano para cristalinos de donante y cinco LIO (uno con filtro UV, dos con filtro violeta y dos con filtro para luz azul) en función de las propiedades de transmisión de los lentes y las características espectrales de la activación de melanopsina. El potencial para la estimulación de melanopsina y la supresión de melatonina se redujo en 0.6-0.7 puntos porcentuales por año de vida debido al amarilleamiento de la lente natural. Los efectos calculados fueron pequeños para los LIO. En consecuencia, se espera que el proceso de envejecimiento natural del cristalino influya en el fotoarrastre del ritmo circadiano, mientras que no se espera que los LIO sean perjudiciales para el ritmo circadiano ${ }^{28}$.

En otro estudio de Bronsted, et al. en 2017, se investigó el efecto de los LIO con filtro de luz azul y los LIO neutros en el fotoarrastre circadiano 1 año después de la cirugía. Los sujetos fueron asignados al azar a la implantación con un LIO neutral de bloqueo UV o un LIO de bloqueo de luz azul. El resultado principal fue la activación de las células ganglionares de la retina intrínsecamente fotosensibles (ipRGC) medida mediante pupilometría cromática. El ritmo circadiano se analizó mediante perfiles de melatonina de 24 horas y actigrafía; esta última también se utilizó para determinar la calidad objetiva del sueño. Un año después de la cirugía, la concentración máxima de melatonina fue de $3.3 \mathrm{pg} / \mathrm{ml}$ (IC 95\%: 2-5.5), el 50\% más baja para los participantes asignados a LIO con filtro azul en comparación con los participantes asignados a LIO neutros. En comparación con los niveles preoperatorios, la respuesta de ipRGC aumentó en el 13.7\% (IC 95\%: 3.2-22.6) 1 año después de la cirugía. La calidad objetiva del sueño también mejoró, ya que el tiempo de vigilia después del inicio del sueño mejoró 5 min (IC 95\%: 1-10) para toda la población, mientras que la eficiencia del sueño aumentó dos puntos porcentuales (IC 95\%: 0.42-3.65) solo en los participantes asignados a LIO con filtro para luz azul. Los LIO con filtro para luz azul mejoraron la respuesta de ipRGC y la calidad del sueño, sin embargo, el efecto sobre la calidad del sueño puede no estar relacionado con el fotoarrastre circadiano ${ }^{29}$.

Kubista, et al. analizaron el efecto de los LIO con filtro para luz azul y compararon el cambio en los niveles diurnos de melatonina después de la implantación en comparación con los LIO sin filtro. Los pacientes fueron aleatorizados para recibir implante de LIO con un filtro para luz azul o LIO transparentes. El análisis de melatonina, la AV, el examen completo con lámpara de hendidura y los hábitos de sueño y un cuestionario de calidad de vida, se realizaron antes y 
1 mes después de la cirugía de catarata. El número promedio de interrupciones del sueño durante la noche aumentó después de la operación en sujetos que recibieron el lente con filtro para luz azul. Los sujetos con lente transparente solo tuvieron un aumento de $<1.0 \mathrm{pg} / \mathrm{ml}$, mientras que los sujetos con filtro para luz azul tuvieron un aumento de $>1.0 \mathrm{pg} / \mathrm{ml}$ de melatonina después de la cirugía. Los sujetos que recibieron LIO con filtro para luz azul tuvieron un aumento del sueño inquieto y más interrupciones del sueño durante la noche relacionadas con niveles elevados de melatonina durante el día ${ }^{30}$.

Leruez, et al. estudiaron el estado de ánimo en pacientes postoperatorios. El propósito del estudio fue determinar si la implantación de LIO con filtro azul afecta el estado de ánimo postoperatorio, induciendo más depresión, en comparación con los pacientes sometidos a implantación con LIO convencionales. Se usó el mismo tipo de LIO en ambos ojos de cada paciente. Los pacientes cognitivamente sanos (un puntaje de examen de estado mini mental superior a 25) fueron evaluados antes y después de la cirugía, utilizando la escala de depresión geriátrica (EDG) de 30 ítems para evaluar los síntomas de depresión. Preoperatoriamente, la $\mathrm{AV}$ y las puntuaciones de EDG fueron comparables en los dos grupos. La puntuación de EDG postoperatoria mejoró en $1.91 \pm 3.10$ puntos en toda la muestra ( $p=0.002$ ), así como en cada subgrupo de pacientes. Tres meses después de la cirugía, el cambio medio en la puntuación de EDG no difirió entre los grupos $(p=0.365)$, al igual que la AV media $(p=0.198)^{31}$.

Zambrowski, et al. compararon los efectos de los LIO amarillos y transparentes sobre el sueño y el estado de ánimo en pacientes de edad avanzada sometidos a cirugía de catarata bilateral. Los sujetos fueron asignados al azar a grupos de LIO amarillos o transparentes. Los pacientes llenaron un diario de sueño, la escala de somnolencia pictórica y el Inventario de depresión de Beck (IDB) 1 semana antes y 8 semanas después del último procedimiento quirúrgico. No se observaron diferencias significativas entre los grupos de LIO amarillos y transparentes con respecto al tiempo de sueño, la latencia del sueño, la duración total del sueño, la calidad del sueño y las puntuaciones de IDB. Pero, sorprendentemente, el número de pacientes cuyo puntaje de IDB aumentó, fue significativamente mayor en el grupo de LIO amarillos, $p=0.02$. Esto sugiere que los pacientes que reciben $\mathrm{LIO}$ amarillos podrían tener un mayor riesgo de desarrollar depresión o, al menos, en lugar de mejorar, el estado de ánimo puede verse alterado en los pacientes que reciben $\mathrm{LIO}$ amarillos. EI uso de LIO amarillos para la cirugía de catarata no afecta significativamente el sueño, pero puede inducir cambios de humor en el envejecimiento. Como una posible explicación, el cambio de humor puede ser la consecuencia de la alteración de los ritmos circadianos que han demostrado tener un impacto significativo en el estado de ánimo ${ }^{32}$.

Kim, et al. evaluaron en 2011 la influencia de los LIO con filtro de luz azul en el grosor de la capa de fibras nerviosas peripapilares de la retina (RNFL). Los pacientes fueron asignados aleatoriamente para recibir un LIO con filtro de luz azul o un LIO transparente. Hubo un aumento significativo del grosor promedio de la RNFL después de la cirugía de catarata en ambos grupos de LIO. No se observaron diferencias significativas en los cambios perioperatorios de las mediciones de RNFL entre los grupos de LIO amarillos y transparentes. El tipo de LIO no afectó las diferencias perioperatorias de las mediciones de grosor de la RNFL $(p=0.002)^{33}$.

Nolan, et al. investigaron si las propiedades de filtrado de luz azul del LIO AcrySof Natural de Alcon implantado durante la cirugía de catarata, afecta la densidad óptica del pigmento macular (DOPM). Los pacientes fueron aleatorizados al LIO acrílico de tres piezas de Alcon AcrySof (controles) o el LIO de Alcon AcrySof Natural implantado en el momento de la cirugía de catarata. Hubo una correlación altamente significativa y positiva entre todas las densidades ópticas de pigmento macular registradas 1 semana antes y después de la cirugía en ojos con un implante de LIO acrílico de tres piezas AcrySof de Alcon $(p<0.01)$ y en aquellos con implante de LIO de Alcon AcrySof Natural (filtro azul) $(p<0.01)$. La densidad óptica promedio de pigmento macular en toda la retina aumentó significativamente con el tiempo (después de 3 meses) en el grupo de LIO de Alcon AcrySof Natural (filtro azul) $(p<0.05)$, pero permaneció estable en el grupo de LIO acrílicos de tres piezas de Alcon AcrySof. Esto proporciona evidencia de que la implantación de un LIO que filtra la luz azul está asociada con el aumento de DOPM. Sin embargo, se necesitan más estudios para evaluar si el aumento de DOPM se asocia con un riesgo reducido de desarrollo y/o progresión de $\mathrm{DMRE}^{34}$.

Las variaciones de grosor macular a lo largo del tiempo en los ojos pseudofáquicos implantados con un LIO transparente en comparación con los ojos contralaterales implantados con un LIO amarillo, se evaluaron en dos sesiones separadas por un intervalo de tiempo de 5 años. Los ojos implantados con un LIO transparente mostraron una reducción estadísticamente significativa 
del grosor macular, $5 \pm 8 \mu \mathrm{m}(\mathrm{p}=0.02)$, y la reducción del grosor foveal fue de $10 \pm 17 \mu \mathrm{m}(p=0.02)$, mayor de lo esperado debido al aumento de la edad. Sin embargo, los ojos implantados con LIO amarillos mantuvieron un grosor macular estable ${ }^{35}$.

En comparación con los anteojos, resumimos los siguientes datos.

Eperjesi, et al. en 2004 determinaron el efecto de las superposiciones de filtros de luz de colores sobre la velocidad de lectura de personas con DMRE que a menudo tienen dificultades con tareas que incluyen lectura, reconocimiento facial y mirar la televisión. La agudeza para leer palabras cercanas se determinó como la palabra más pequeña que se podía leer a $25 \mathrm{~cm}$ utilizando la corrección refractiva óptima. La prueba de velocidad de lectura mostró que la velocidad de lectura no estaba relacionada con el color del filtro $(p=0.08)$. Las superposiciones de filtros de luz de colores no mejoraron la velocidad de lectura; es poco probable que la aberración cromática longitudinal afecte la lectura en la DMRE ${ }^{36}$.

Leung, et al. analizaron el impacto de los anteojos con filtro azul en el desempeño visual y la calidad del sueño. Se evaluaron «BlueControl» (Hoya, Japón), «BlueProtect» (Zeiss, Alemania), "Crizal Prevencia» (Essilor, Francia), "StressFree» y «Noflex» (Swiss Lens, Hong Kong). Los cambios relativos en fototoxicidad, sensibilidad escotópica y supresión de melatonina se calcularon en función de sus transmisiones espectrales medidas por un espectrofotómetro. El desempeño clínico de dos anteojos con filtro de luz azul se comparó con anteojos transparentes regulares que sirvieron como control. Se midió la SC en condiciones estándar y de glare, y la discriminación de colores. Después de cada periodo de uso mensual, se evaluaron de forma subjetiva mediante un cuestionario el desempeño del lente de los participantes, la calidad de la visión nocturna y la calidad del sueño. Todos los anteojos con filtro para luz azul analizados redujeron teóricamente la fototoxicidad de un 10.6 a $23.6 \%$. Aunque el uso de este filtro también disminuyó la sensibilidad escotópica entre un 2.4 y un $9.6 \%$, y la supresión de melatonina entre un 5.8 y un $15.0 \%$, más del $70 \%$ de los participantes no pudieron detectar estos cambios ópticos. Sus pruebas clínicas tampoco revelaron una disminución significativa en la SC. Estos lentes pueden servir como una opción suplementaria para proteger la retina del peligro potencial de la luz azul ${ }^{37}$.

Bonnin, et al. compararon el efecto de los nuevos anteojos de sol con certificación de seguridad retiniana
(RSC) y los anteojos de sol tradicionales sobre la función visual. Los anteojos de sol se caracterizaron por medio del espectrofotómetro Humphrey, y la principal diferencia entre los anteojos de sol nuevos con RSC y los tradicionales está en la absorbancia selectiva de las longitudes de onda cortas. Los aspectos de la función visual evaluados fueron: AV, estereoacuidad (SA), percepción de colores y FSC. Los valores de AV y SA con el filtro solar con RSC fueron similares a los obtenidos sin el filtro; sin embargo, con el filtro solar tradicional, ambas variables disminuyen significativamente. La discriminación de colores disminuye con ambos filtros, con una pérdida muy superior con el filtro tradicional $(45 \%)$ en comparación con el filtro con RSC (5\%). En consecuencia, estos lentes mejoran la visibilidad y la comodidad al tiempo que mantienen la protección solar y, al mismo tiempo, optimizan la AV, la SA y la FSC y mantienen la percepción del colores ${ }^{38}$.

Ide, et al., estudiaron el efecto de bloquear la luz de longitud de onda corta sobre la frecuencia de parpadeo de fusión crítica (CFF). La población del estudio se dividió en tres grupos según el tipo de anteojos utilizados durante el estudio: lentes con filtro para luz azul (lente 1, tasa de bloqueo alta), lentes con filtro para luz azul (lente 2, tasa de bloqueo baja) o lentes transparentes (lente 3 , control). Antes y después de realizar las tareas experimentales, se evaluó la fatiga visual calculando la CFF y evaluando las respuestas a un cuestionario de 13 ítems sobre quejas visuales (ojos secos, ojos irritados, dificultad para reenfocar los ojos, fotofobia, picazón en los ojos, tensión/fatiga ocular, estrés mental, somnolencia al trabajar, dolor de cuello hombro/espalda/cintura). El tipo de lente utilizado afectó significativamente la CFF; sin embargo, las respuestas a los cuestionarios subjetivos no difirieron significativamente entre los grupos. Solo dos de los 13 ítems de preguntas (dolor de cuello/hombro/espalda/cintura y tensión/fatiga ocular) mostraron una diferencia estadística entre la transparencia de los lentes. Cuanto mayor sea el efecto de bloqueo de la lente, menor será la reducción de la CFF, lo que sugiere que bloquear la luz de onda corta puede reducir la fatiga ocular $^{39}$.

Kaido, et al. investigaron si la supresión de la luz azul puede mejorar la función visual en pacientes con ojo seco con un tiempo de ruptura de la película lagrimal (TRPL) corto. Veintidós pacientes con TRPL corto y 18 controles sanos se sometieron a exámenes de AV con y sin anteojos, con un $50 \%$ de capacidad de bloqueo de la luz azul. Los parámetros de AV funcionales fueron $A V$ de referencia, AV funcional y relación de 
mantenimiento visual. Los valores medios basales de AV funcional y la relación de mantenimiento visual fueron significativamente peores en los pacientes con TRPL corto que en los controles $(p<0.05)$, mientras que no se observaron diferencias significativas de la AV basal. Los pacientes con TRPL corto tuvieron una mejoría significativa en la media de AV funcional y la relación de mantenimiento visual mientras usaban anteojos $(p<0.05)$, mientras que no hubo cambios significativos con y sin anteojos en el grupo control $(p>0.05)$. Proteger los ojos de la luz azul de onda corta puede ayudar a mejorar la discapacidad visual asociada con la inestabilidad lagrimal en pacientes con TBUT corto ${ }^{40}$.

En 2010, Eperjesi, et al. realizaron un estudio para evaluar los efectos de tres filtros amarillos comerciales sobre la AV y la SC medidos con la prueba de Pelli-Robson (con y sin glare) y la lectura medida con tablas MNRead (sin glare) en condiciones de dispersión de luz. Se observaron diferencias estadísticamente significativas entre el efecto general del glare y entre los filtros fotocromáticos de Corning (CPF) para la AV y la SC. Se observó una disminución gradual de la AV, la SC y la lectura con el aumento de la absorción de $\mathrm{CPF}^{41}$. Además, Mahjoob, et al. evaluaron el efecto del filtro amarillo sobre la AV y SC en condiciones de dispersión de luz en diferentes grupos de edad. Su hipótesis era que el filtro amarillo podría cambiar el desempeño visual analizado y tener resultados diferentes en adultos mayores y más jóvenes. En general, no hubo diferencias estadísticamente significativas de la AV con y sin glare $(p=0.083)$, tampoco se encontró un efecto significativo del filtro amarillo en la AV de los pacientes bajo glare $(p=0.564)$. Por otro lado, hubo una diferencia significativa entre la media de SC con y sin deslumbramiento $(p=0.000)$ y se encontró un efecto significativo del filtro amarillo en la AV de los pacientes bajo glare $(p=0.000)$. La SC en todos los grupos de edad en condiciones de glare fue significativamente menor que la SC sin glare. Los resultados mostraron que los efectos de dispersión de la luz aumentaron con la edad y que podrían reducirse con el uso del filtro amarillo ${ }^{42}$.

Burkhart, et al. en 2009 asignaron al azar a 20 adultos a utilizar anteojos de seguridad con filtro azul (ámbar) o filtro amarillo (solo bloqueo UV) durante 3 horas antes de dormir. Los participantes llenaron diarios de sueño durante una evaluación inicial de una semana y dos semanas de uso de anteojos. Las medidas de resultados fueron subjetivas: cambio en la calidad general del sueño y efecto positivo/negativo. El grupo de lentes de color ámbar experimentó una mejora significativa $(p<0.001)$ en la calidad del sueño en relación con el grupo control, y un efecto positivo $(p=0.005)$. El estado de ánimo también mejoró significativamente en relación con los controles ${ }^{43}$. Además del tema del estado de ánimo, Sasseville, et al. en 2006 analizaron la hipótesis de que eliminar la parte azul del espectro de luz con lentes de color naranja (filtro para luz azul) evitaría la supresión de la melatonina inducida por la luz. Se comparó el cambio en la cantidad de melatonina salival con una basal de melatonina obtenida la noche anterior. Aunque ambos lentes transmitieron la misma iluminación, la prueba ANOVA encontró un aumento no significativo del 6\% (IC 95\%: 20\%-9\%) con el lente naranja, mientras que se observó una reducción significativa $(p<0.05)$ del 46\% (IC 95\%: 35-57\%) con el lente gris. Los bloqueadores de la luz azul representan un medio elegante para prevenir la supresión de melatonina inducida por la luz ${ }^{44}$.

Investigadores de la Facultad de Medicina de la Universidad de Keio investigaron la calidad del sueño y los niveles de melatonina en adultos que usaban anteojos con filtro para luz azul o anteojos control 2 horas antes de dormir mientras usaban un dispositivo portátil autoluminiscente, y evaluaron la calidad visual para los dos tipos de anteojos, monitoreando cada ciclo de sueño/ vigilia del paciente con un registrador de micromovimiento, y haciendo un cuestionario validado (que incorporaba el índice de calidad del sueño de Pittsburgh y la escala de sueño Karolinska) antes y durante el experimento. La secreción de melatonina durante la noche fue significativamente mayor después de usar los anteojos con filtro para luz azul $(p<0.05)$ en comparación con los anteojos control. La eficacia del sueño y la latencia del sueño fueron significativamente superiores para los usuarios de anteojos con filtro de luz azul $(p<0.05$ para ambos), y este grupo informó una mayor somnolencia durante el uso de dispositivos portátiles en comparación con los que usaron los anteojos control ${ }^{45}$.

Otro estudio con anteojos y dispositivos electrónicos fue realizado por Lin, et al. en 2017. Su propósito era determinar si los sujetos que usan anteojos que bloquean la longitud de onda corta durante las tareas de la computadora, exhiben menos fatiga visual e informan menos síntomas de incomodidad visual que los sujetos que usan anteojos transparentes. Un calificador enmascarado midió la frecuencia de parpadeo de fusión crítica (CFF) como una métrica de fatiga ocular. Encontraron que el cambio de CFF después de la tarea de la computadora fue significativamente más positivo (menos fatiga ocular) en el grupo de bloqueo alto versus el grupo $\sin$ bloqueo $(p=0.027)$. Además, la 
asignación aleatoria al grupo de bloqueo alto, pero no al grupo de bloqueo bajo, predijo un cambio más positivo de la CFF (es decir, menos fatiga ocular) después de realizar tareas en la computadora $(p=0.002)$. Además, los sujetos que utilizaron anteojos con bloqueo alto informaron significativamente menos dolor alrededor/dentro del ojo ( $p=0.0063$ ), menos sensación de pesadez ocular $(p=0.0189)$ y menos sensación de picazón en los ojos $(p=0.0043)$ después de realizar tareas en la computadora, en comparación con sujetos que no usaron lentes de bloqueo alto. Los resultados apoyan la hipótesis de que los anteojos que bloquean la luz de longitud de onda corta pueden reducir la fatiga ocular asociada con el uso de la computadora con base en una correlación fisiológica de la fatiga ocular y en los informes de los sujetos de síntomas típicamente asociados con la fatiga visual; sin embargo, parece que la frecuencia de parpadeo es independiente de la exposición a la luz azul|46.

Colombo, et al. evaluaron los parámetros visuales funcionales utilizando lentes fotocromáticos y selectivos con filtro para luz azul en pacientes con escotoma central (grupo 1) o periférico (grupo 2) debido a enfermedades de la retina. La AV mejor corregida en negro sobre blanco (BW-BCVA), la AV mejor corregida en blanco sobre negro (WB-BCVA), SC de Mars y una prueba de glare (GT) se realizaron en todos los pacientes. Se compararon los resultados de la prueba con el filtro azul-violeta, un filtro amarillo de paso corto y sin filtros. La media de BW-BCVA aumentó de $0.30 \pm 0.20$ a $0.36 \pm 0.21$ decimales en el grupo 1 y de $0.44 \pm 0.22$ a $0.51 \pm 0.23$ decimales en el grupo $2(p<0.0001)$. La media de WB-BCVA aumentó de $0.31 \pm 0.19$ a $0.38 \pm$ 0.23 decimales en el grupo 1 y de $0.46 \pm 0.20$ a 0.56 \pm 0.22 decimales en el grupo $2(p<0.0001)$. El recuento de letras para la prueba de SC aumentó de $26.7 \pm$ 7.9 a $30.06 \pm 7.8$ en el grupo $1(p=0.0005)$ y de 31.5 \pm 7.6 a $33.72 \pm 7.3$ en el grupo $2(p=0.031)$. La GT disminuyó significativamente: el recuento de letras aumentó de $20.93 \pm 5.42$ a $22.82 \pm 4.93$ en el grupo 1 $(p<0.0001)$ y de $24.15 \pm 5.5$ a $25.97 \pm 4.7$ en el grupo $2(p<0.0001)$. Se registraron puntuaciones más altas con el filtro azul en comparación con el filtro amarillo para todas las pruebas $(p<0.05)$. No se pudieron detectar diferencias significativas en los resultados de las pruebas entre el filtro amarillo y la condición sin filtro. El uso de una combinación de lente fotocromático con un filtro selectivo para luz azul-violeta mostró un beneficio funcional en todos los pacientes evaluados ${ }^{47}$.

La principal fortaleza de este análisis sistemático es la evaluación rigurosa de la calidad de los estudios incluidos y la comparación entre los LIO y otros dispositivos oftalmológicos. Una limitación de nuestro estudio es la inclusión de estudios publicados únicamente y, por lo tanto, no se excluye el sesgo de publicación.

\section{Conclusiones}

Con respecto a los LIO que bloquean la luz azul, ningún estudio muestra efectos nocivos sobre el desempeño visual, incluida la AV, la SC y la percepción del color, incluso en ojos con enfermedad macular. Hubo una mejora general en la SC bajo condiciones mesópicas y fotópicas. Los pacientes informaron menos molestias por glare, y la percepción del color se informó como «más cómoda» con estos LIO.

La ventaja potencial de los LIO con color para proporcionar protección a las células maculares sigue sin estar clara. El estado de ánimo postoperatorio mejora en la mayoría de los pacientes después de la cirugía de catarata, y esto no está relacionado con el filtro del LIO. Los LIO que bloquean la luz azul pueden mejorar la calidad del sueño.

Hay una falta de estudios clínicos de calidad que muestren los beneficios de los anteojos con estos filtros en comparación con los LIO. Todos los anteojos con filtro de luz azul probados redujeron teóricamente la fototoxicidad y pueden servir como una opción adicional para proteger la retina de un posible riesgo por la luz azul.

Los dispositivos emisores de longitud de onda corta contribuyen a la fatiga visual. De hecho, el uso prolongado de estos dispositivos puede dar lugar a una constelación de síntomas, que ahora se reconocen como síndrome de visión digital. El bloqueo de la luz de longitud de onda corta puede reducir la fatiga ocular y puede ayudar a mejorar la discapacidad visual asociada con la inestabilidad lagrimal en pacientes con ojo seco. Con respecto al síndrome de visión digital que induce fatiga visual, cefalea, molestias oculares, ojo seco, diplopía y visión borrosa, es una afección multifactorial con causas muy diferentes como anomalías de la película lagrimal, enfermedades reumatológicas sistémicas, trastornos oculomotores e incluso errores refractivos no corregidos. Es por eso que delimitar el papel de la luz azul en este síndrome es un poco difícil.

Este tipo de lentes muestra un ligero aumento en la SC, pero con un impacto clínico muy bajo. Algunos estudios sugieren una mejora en la calidad del sueño con un aumento no significativo en los niveles séricos de melatonina. 
El daño retiniano inducido por LED en modelos animales muestra dependencia de la longitud de onda. Desde el descubrimiento de los riesgos de la luz azul, los lentes con filtro de luz azul se han considerado como una opción viable para la protección de la retina, pero el impacto real de este procedimiento no se ha demostrado definitivamente. Aunque la exposición humana a la luz de longitud de onda corta generalmente es crónica y subumbral en lugar de aguda y supraumbral, como lo es para la mayoría de estos modelos animales, los estudios analizados implican que la luz de longitud de onda corta es patológica. Aunque el aumento de la edad, la genética y el tabaquismo son factores de riesgo bien establecidos para la DMRE, todavía no está claro si otros aspectos, incluida la exposición a largo plazo a la luz de onda corta, podrían contribuir al desarrollo de la enfermedad.

En la literatura médica actual, se identificaron pocos documentos que informaran los efectos de los anteojos que bloquean la luz azul en la salud de la retina, y todos concluyen que la irradiación de estos dispositivos no alcanza los límites internacionales de exposición para daño de la retina, incluso con la visualización prolongada. Con estas consideraciones, un uso más amplio de anteojos con filtro de luz azul, especialmente por las personas mayores con un mayor riesgo de progresión de DMRE, podría desempeñar un papel complementario en la preservación visual y podría ser una opción para una mayor protección contra las enfermedades de la retina.

Un aspecto clave de los filtros de bloqueo de luz azul es encontrar el equilibrio entre reducir efectivamente los riesgos de la luz azul sin comprometer las funciones visuales esenciales de la vida diaria. Los estudios a largo plazo bien diseñados podrían revelar qué espectro de luz visible se debe filtrar para minimizar los riesgos para la salud, permitiendo una transmisión suficiente de luz azul para permitir realizar las funciones fisiológicas normales, además de definir cuál es el papel real de la luz azul en la patogenia de la enfermedad retiniana, tanto degenerativa como distrófica. Los estudios futuros no solo explorarán los efectos de la exposición crónica a la luz azul, sino que también identificarán las características de la luz azul que producen estos efectos tóxicos.

Es difícil generalizar basándose solo en nuestros resultados; sin embargo, los autores esperan que con los resultados de esta revisión sistemática pueda ser útil para futuros estudios clínicos para mejorar la metodología y el análisis estadístico para abordar mejor los beneficios de estos filtros en la población general.

\section{Conflicto de intereses}

Los autores no tienen ningún conflicto de intereses que revelar con respecto a esta revisión.

\section{Responsabilidades éticas}

Protección de personas y animales. Los autores declaran que para esta investigación no se han realizado experimentos en seres humanos ni en animales.

Confidencialidad de los datos. Los autores declaran que en este artículo no aparecen datos de pacientes.

Derecho a la privacidad y consentimiento informado. Los autores declaran que en este artículo no aparecen datos de pacientes.

\section{Bibliografía}

1. Rezai KA, Gasyna E, Seagle B-LL, Norris JR, Rezaei KA. AcrySof Natural filter decreases blue light-induced apoptosis in human retinal pigment epithelium. Graefes Arch Clin Exp Ophthalmol. 2008;246(5):671-6.

2. Tosini G, Ferguson I, Tsubota K. Effects of blue light on the circadian system and eye physiology. Mol Vis. 2016;22:61-72.

3. Kernt M, Walch A, Neubauer AS, Hirneiss C, Haritoglou MD C, Ulbig MW, et al. Filtering blue light reduces light-induced oxidative stress, senescence and accumulation of extracellular matrix proteins in human retinal pigment epithelium cells. Clin Experiment Ophthalmol. 2012;40(1):e87-e97.

4. Zhao Z-C, Zhou Y, Tan G, Li J. Research progress about the effect and prevention of blue light on eyes. Int J Ophthalmol. 2018;11(12):1999-2003.

5. Algvere PV, Marshall J, Seregard S. Age-related maculopathy and the impact of blue light hazard. Acta Ophthalmol Scand. 2006;84(1):4-15.

6. Langagergaard U, Ganer HJ, Baggesen K. Age-related macular degeneration: filter lenses help in certain situations. Acta Ophthalmol Scand. 2003;81(5):455-8.

7. Moon J, Yun J, Yoon YD, Park S-I, Seo Y-J, Park WS, et al. Blue light effect on retinal pigment epithelial cells by display devices. Integr Biol Quant Biosci Nano Macro. 2017;9(5):436-43.

8. PR Newswire. The "Greening of America» and Dependence on Technology May Damage Eyes and Impact Health, According to BluTech $\circledast$ Lenses. EYE-Solut-TECH. 2014 [citado 19 de febrero de 2019]; Disponible en: https://www.prnewswire.com/news-releases/the-greening-of-america-and-dependence-on-technology-may-damage-eyes-and-impact-health-according-to-blutech-lenses-241525271.html

9. Glazer-Hockstein C, Dunaief JL. Could blue light-blocking lenses decrease the risk of age-related macular degeneration?. Retina Phila Pa. 2006;26(1):1-4.

10. Cuthbertson FM, Peirson SN, Wulff K, Foster RG, Downes SM. Blue light-filtering intraocular lenses: review of potential benefits and side effects. J Cataract Refract Surg. 2009;35(7):1281-97.

11. Downes SM. Ultraviolet or blue-filtering intraocular lenses: what is the evidence?. Eye Lond. 2016;30(2):215-21.

12. Downie LE, Busija L, Keller PR. Blue-light filtering intraocular lenses (IOLs) for protecting macular health. Cochrane Database Syst Rev. 2018;5:CD011977.

13. Davison JA, Patel AS, Cunha JP, Schwiegerling J, Muftuoglu O. Recent studies provide an updated clinical perspective on blue light-filtering IOLs. Graefes Arch Clin Exp Ophthalmol Albrecht Von Graefes Arch Klin Exp Ophthalmol. 2011;249(7):957-68.

14. Lawrenson JG, Hull CC, Downie LE. The effect of blue-light blocking spectacle lenses on visual performance, macular health and the sleepwake cycle: a systematic review of the literature. Ophthalmic Physiol Opt J Br Coll Ophthalmic Opt Optom. 2017;37(6):644-54.

15. Eperjesi F, Fowler CW, Evans BJW. Do tinted lenses or filters improve visual performance in low vision? A review of the literature. Ophthalmic Physiol Opt. 2002;22(1):68-77.

16. Augustin AJ. The physiology of scotopic vision, contrast vision, color vision, and circadian rhythmicity: can these parameters be influenced by blue-light-filter lenses?. Retina Phila Pa. 2008;28(9):1179-87. 
17. Henderson BA, Grimes KJ. Blue-blocking IOLs: a complete review of the literature. Surv Ophthalmol. 2010;55(3):284-9.

18. Hovis JK, Lovasik JV, Cullen AP, Kothe AC. Physical characteristics and perceptual effects of «blue-blocking» lenses. Optom Vis Sci. 1989;66(10):682-9.

19. Owczarek G, Gralewicz G, Skuza N, Jurowski P. Light transmission through intraocular lenses with or without yellow chromophore (blue light filter) and its potential influence on functional vision in everyday environmental conditions. Int J Occup Saf Ergon. 2016;22(1):66-70.

20. Leibovitch I, Lai T, Porter N, Pietris G, Newland H, Selva D. Visual outcomes with the yellow intraocular lens. Acta Ophthalmol Scand. 2006;84(1):95-9.

21. Rodríguez-Galietero A, Montés-Micó R, Muñoz G, Albarrán-Diego C. Blue-light filtering intraocular lens in patients with diabetes: contrast sensitivity and chromatic discrimination. J Cataract Refract Surg. 2005;31(11):2088-92.

22. Hammond BR Jr, Renzi LM, Sachak S, Brint SF. Contralateral comparison of blue-filtering and non-blue-filtering intraocular lenses: glare disability, heterochromatic contrast, and photostress recovery. Clin Ophthalmol Auckl NZ. 2010:4:1465-73.

23. Da Costa MF, Júnior AP, Lottenberg CL, Castro LC, Ventura DF. Psychophysical Measurements of Luminance Contrast Sensitivity and Color Discrimination with Transparent and Blue-Light Filter Intraocular Lenses. Ophthalmol Ther. 2017;6(2):301-12.

24. Kara-Junior N, Espindola RF, Gomes BAF, Ventura B, Smadja D, Santhiago MR. Effects of blue light-filtering intraocular lenses on the macula, contrast sensitivity, and color vision after a long-term follow-up. J Cataract Refract Surg. 2011;37(12):2115-9.

25. Lavric A, Pompe MT. Do blue-light filtering intraocular lenses affect visua function?. Optom Vis Sci. 2014:91(11):1348-54

26. Mokuno K, Asami T, Nonobe N, Ito H, Fujiwara K, Terasaki H. Effect of blue light-filtering intraocular lens on color vision in patients with macular diseases after vitrectomy. Int Ophthalmol. 2016;36(6):829-39.

27. Kiser AK, Deschler EK, Dagnelie G. Visual function and performance with blue-light blocking filters in age-related macular degeneration. Clin Experiment Ophthalmol. 2008;36(6):514-20.

28. Brøndsted $A E$, Lundeman JH, Kessel L. Short wavelength light filtering by the natural human lens and IOLs implications for entrainment of circadian rhythm. Acta Ophthalmol (Copenh). 2013;91(1):52-7.

29. Brøndsted AE, Haargaard B, Sander B, Lund-Andersen $H$, Jennum $P$ Kessel $\mathrm{L}$. The effect of blue-blocking and neutral intraocular lenses on circadian photoentrainment and sleep one year after cataract surgery. Acta Ophthalmol (Copenh). 2017;95(4):344-51.

30. Kubista KE, Brunner S, Glittenberg CG, Hochwarter A, Binder S. Influence of blue-light-filtering intraocular lenses on daytime levels of melatonin (BluMel-Study). Spektrum Augenheilkd. 2013;27(4):176-80.

31. Leruez S, Annweiler C, Gohier B, Beauchet O, Ebran J-M, Gohier P et al. Blue light-filtering intraocular lenses and post-operative mood: a pilot clinical study. Int Ophthalmol. 2015;35(2):249-56.

32. Zambrowski O, Tavernier E, Souied EH, Desmidt T, Le Gouge A, Bellicaud D, et al. Sleep and mood changes in advanced age after blue-blocking (yellow) intra ocular lens (IOLs) implantation during cataract surgical treatment: a randomized controlled trial. Aging Ment Health. 2018;22(10):1351-6.
33. Kim JH, Kim NR, Lee ES, Rho S, Kang SY, Kim CY. Influence of blue light-filtering intraocular lenses on retinal nerve fiber layer measurements by spectral-domain optical coherence tomography. Curr Eye Res. 2011;36(10):937-42.

34. Nolan JM, O'Reilly P, Loughman J, Stack J, Loane E, Connolly E, et al. Augmentation of macular pigment following implantation of blue light-filtering intraocular lenses at the time of cataract surgery. Invest Ophthalmol Vis Sci. 2009;50(10):4777-85.

35. Chamorro E, Bonnin-Arias $\mathrm{C}$, Pérez-Carrasco MJ, Álvarez-Rementería L, Villa-Collar C, Armadá-Maresca F, et al. Espesor macular medido con tomografía de coherencia óptica en ojos pseudoafáquicos con implante amarillo vs. transparente. Arch Soc Esp Oftalmol. 2014;89(4):136-42.

36. Eperjesi F, Fowler CW, Evans BJW. The effects of coloured light filter overlays on reading rates in age-related macular degeneration. Acta Ophthalmol Scand. 2004;82(6):695-700.

37. Leung TW, Li RW-H, Kee C-S. Blue-Light Filtering Spectacle Lenses: Optical and Clinical Performances. PLoS One. 2017;12(1):e0169114.

38. Bonnin Arias C, Aguirre Vilacoro V, Chamorro Gutiérrez E, Sánchez Ramos C. Nuevos lentes solares con certificado de seguridad retiniana: análisis de la función visual mediante la valoración de agudeza visual y estereoscópica, discriminación del color y sensibilidad al contraste. Ciencia \& tecnología para la salud visual y ocular. 2015;13(1):123-36.

39. Ide T, Toda I, Miki E, Tsubota K. Effect of Blue Light-Reducing Eye Glasses on Critical Flicker Frequency. Asia-Pac J Ophthalmol Phila Pa. 2015;4(2):80-5.

40. Kaido M, Toda I, Oobayashi T, Kawashima M, Katada Y, Tsubota K Reducing Short-Wavelength Blue Light in Dry Eye Patients with Unstable Tear Film Improves Performance on Tests of Visual Acuity. PLoS One. 2016;11(4):e0152936.

41. Eperjesi F, Agelis LE. Effects of yellow filters on visual acuity, contrast sensitivity and reading under conditions of forward light scatter. Graefes Arch Clin Exp Ophthalmol Albrecht Von Graefes Arch Klin Exp Ophthalmol. 2011;249(5):709-14.

42. Mahjoob M, Heydarian S, Koochi S. Effect of yellow filter on visual acuity and contrast sensitivity under glare condition among different age groups. Int Ophthalmol. 2016;36(4):509-14.

43. Burkhart K, Phelps JR. Amber lenses to block blue light and improve sleep: a randomized trial. Chronobiol Int. 2009;26(8):1602-12.

44. Sasseville A, Paquet N, Sévigny J, Hébert M. Blue blocker glasses impede the capacity of bright light to suppress melatonin production. $\mathrm{J} \mathrm{Pi}$ neal Res. 2006;41(1):73-8.

45. Ayaki M, Hattori A, Maruyama Y, Nakano M, Yoshimura M, Kitazawa M, et al. Protective effect of blue-light shield eyewear for adults against light pollution from self-luminous devices used at night. Chronobiol Int. 2016;33(1):134-9.

46. Lin JB, Gerratt BW, Bassi CJ, Apte RS. Short-Wavelength Light-Blocking Eyeglasses Attenuate Symptoms of Eye Fatigue. Invest Ophthalmol Vis Sci. 2017;58(1):442-7.

47. Colombo L, Melardi E, Ferri P, Montesano G, Samir Attaalla S, Patelli F, et al. Visual function improvement using photocromic and selective blue-violet light filtering spectacle lenses in patients affected by retinal diseases. BMC Ophthalmol. 2017;17(1):149. 